\title{
UNA VUELTA DE TUERCA A LOS TEMAS SOBERANOS Y DE PACTO EN LAS RELACIONES MAPUCHE-WINGKA A LA LUZ DE LA OBRA DE TOM DILLEHAY
}

\author{
Rolf Foerster G. ${ }^{1}$ y José A. Isla M. ${ }^{1}$
}

¿Cómo entender el "concepto y la práctica de la soberanía araucana" desde el siglo XVI hasta el presente? El artículo de Dillehay dedicado a "ensayar" una respuesta a esta gran pregunta resulta desafiante y provocador, al menos, por dos razones. Primero, porque responder a este dilema implica hacerse cargo de una reflexión de larga data que comenzó a fines del siglo XVI cuando la guerra en la Araucanía llegó a un estado de "irresolución" permanente, creando un contexto social único en la América Hispana. Existe, desde luego, una discusión temprana que abordó esta cuestión en "tiempo real" (cuyos argumentos más relevantes se encuentran en La Araucana de Ercilla, Desengaño y Reparo de González de Nájera, El Cautiverio Feliz de Pineda y Bascuñán y el Flandes Indiano de Rosales). Asimismo, hay un debate contemporáneo que vuelve acerca de esta misma cuestión en términos históricos y antropológicos (entre los textos más importantes están Los vencedores de Boccara, Historia de los antiguos mapuches del sur de Bengoa y Monumentos, imperios y resistencia en los Andes de Dillehay). Es necesario hacerse cargo de ambas discusiones para avanzar una respuesta significativa. Segundo: hay que recordar que no se trata de un problema exclusivamente histórico. La pregunta respecto del "concepto y la práctica de la soberanía araucana" implica abordar una de las claves para la comprensión del conflicto mapuche en las últimas décadas, así como de las actuales movilizaciones y demandas de las organizaciones mapuche.

Profundicemos, en primer lugar, en las consecuencias que el artículo que comentamos sostiene respecto de aquello que en el párrafo anterior llamamos la discusión histórica y antropológica contemporánea del tema. La hipótesis de Dillehay plantea una continuidad en el modo en cómo se ejerce (y expresa) la soberanía en el mundo mapuche. Se trata de una forma distinta a la occidental, por lo que entre ambas se verifica una suerte de ontological rupture y sobre esto hay consenso en el debate etnohistórico y etnográfico. Sin embargo, la tesis de la pura continuidad en el procesamiento de la soberanía lo distancia de las ideas de Boccara respecto de la emergencia de un proceso de etnogénesis mapuche, postulando en su lugar la tesis de una "etnomorfosis", entendida como el paso de un "estado de etnicidad a otro". Este alejamiento de la noción de etnogénesis resulta una fuerte provocación al debate, porque la obra de Boccara es la más novedosa, sistemática y fundada que tenemos, hasta ahora, para comprender la transformación mapuche de los siglos XVII y $\mathrm{XVIII}^{1}$. Creemos, por las consecuencias que se derivan, que no debiera bastar simplemente un párrafo para proceder a un descarte, sin producir el diálogo crítico que merece la obra de Boccara. Esta réplica debe pasar necesariamente por la cuestión de los Parlamentos coloniales, la institución por la cual indígenas e hispanos negociaron la cuestión de la soberanía. Es en los Parlamentos donde se cristaliza una lógica de la soberanía exógena respecto de los indígenas, y en alguna medida también respecto de los hispanos. Acerca del fondo de la lógica de los Parlamentos emerge una etnicidad mapuche que se plantea como contraparte a los wingka. Fuera del entramado político de los Parlamentos, en la pura identidad de las "comunidades", esa etnicidad carece de sentido (resulta entonces "insignificante"). Este otro espacio es el mundo de las personas "normales" (los reche) constituidas desde una operación distinta a la lógica étnica. Es posible cuestionar el carácter de "salto" que Boccara propone para comprender el desplazamiento de perspectiva que va de la categoría reche a la categoría mapuche; pero para entender las relaciones interétnicas que se verifican en el espacio de "frontera" mapuche/wingka constituido a partir del siglo XVII, resulta muy difícil ignorar la transformación propuesta por este autor.

\footnotetext{
1 Departamento de Antropología, Facultad de Ciencias Sociales, Universidad de Chile, Santiago, Chile. rfoerster@uchile.cl; jaisla@gmail.com
} 
Para Dillehay la armadura social de la soberanía mapuche conjuga una local centralization con una regional non-centralization. Coincidimos en lo grueso con esta apreciación ${ }^{2}$. En otras palabras, entre los mapuche lo local no pierde su centralidad al formar parte de una región no centralizada. La vinculación de la "parte" con el "todo" no es metafórica, sino que toma la forma de una sinécdoque. La parte puede designar al todo o el todo puede designar a las partes, dependiendo de la perspectiva asumida por el interlocutor. Esta conjugación perspectivista, un rasgo claro y reconocible entre los mapuche, apunta más hacia una forma social del tipo Sociedad-contrael-Estado (a la Clastres) que al modelo político andino en el que Dillehay cree ver involucrados a los mapuche, al interior, de un proceso protoestatal que despierta muchas dudas. La confirmación de esta distancia clastreana puede encontrarse en otro rasgo que Boccara pone de relieve, pero que Dillehay minimiza: la omnipresencia paradójica de la guerra. Como ya lo constatara el muy notable informe del maestre de campo Alonso González de Nájera, la emergencia de los pactos con los indígenas no sustituye a la guerra. Tal vez es en este aspecto donde mejor se aprecia la ontological rupture que plantea el modo en cómo se ejerce (y expresa) la soberanía en el mundo mapuche.

La colonización hispánica funcionó en América bajo el paradigma de lo que hoy se conoce como settler colonialism (un proceso irreversible, a no ser por "limpieza étnica"). Ahora bien, en el caso del "Reino de Chile" ese proceso se dio efectivamente en Chile Central, pero fracasó en la zona de la Araucanía. La evidencia para todos (desde el rey a los gobernadores, pasando por las órdenes religiosas, en especial de los jesuitas) fue el alzamiento general (de Arauco a Osorno) ocasionado por Curalaba a fines del siglo XVI. Para los historiadores chilenos ese "acontecimiento" puso fin a la "conquista" y dio inicio a la "Colonia" (Barros Arana).

La "solución" para la guerra en la Araucanía fueron los Parlamentos, donde se aprecia que guerra y política son procesos reversibles y vinculados. Esta situación permite dos enfoques que están presentes en la reflexión de Dillehay, por un lado, el tema de la guerra (o resistencia):

Mapuche successfully resisted the Spanish conquest for more than 250 years by forming a confederated proto-state or polity comprised of partner domains (i.e., Catiray, Arauco, Tucapel, Puren) and different ethnic groups.

Y por otro, el de la política:

It is important that royal opinion in Spain during this period recognized another imperial sovereign, an indigenous one in the Americas, despite the fact that the Spaniards saw the "indios" as pagan, barbarian, and idolatrous. In fact, the Araucanians constitute the only indigenous ethnic group in the Americas that concluded a formal treaty and established political boundary with the Spanish Crown.

Lo que Dillehay no problematiza de ese proceso es lo que podemos valorar del enfoque de Boccara: la política de los Parlamentos es escenario y motor de la etnogénesis ${ }^{3}$. Recordemos que esta ya es la tesis de Vicuña Mackenna (1869):

el renombrado Luis de Valdivia, había sido causa en 1612 de la funesta guerra defensiva, que hizo de la manada de Arauco una nación con fuero, con derecho, con parlamentos, con tributos pagados por nosotros, ¿qué digo? con fronteras con las que nos separaban de otras naciones.

Desde nuestra perspectiva y siguiendo los trabajos de Leonardo León en la década de 1990 (León 1992), vemos en esta escena política hispanomapuche una suerte de middleground o "Pacto colonial". Así los mapuches dejaron de ver a los hispanos solo como outsiders and invaders, pasando a ocupar un lugar permanente como "otros" incluidos en el mapa de la sociabilidad. En el proceso de etnogénesis la construcción de la propia identidad requiere de la presencia permanente del otro.

Lo que Dillehay no ve es lo que propusieron los "estudios fronterizos": des-construir el "mito de la guerra" para tomar en serio a toda una pléyade de figuras fronterizas como los "capitanes de amigo" o los "comisario de naciones", "los caciques gobernadores" o incluso los mismos "butalmapus". Lamentablemente esta innovación de los "estudios fronterizos" derivó en una sobrevaloración de la frontera, olvidando la dimensión interétnica de las relaciones sociales que fueron la materia prima 
de esta frontera. La clave está en que, a partir del siglo XVII, la puesta en escena de la dimensión soberana mapuche se hace inseparable de una suerte de "Pacto colonial": una de las caras de la legitimidad de los caciques pasaba por sus vínculos con figuras como los "capitanes de amigo" o los "comisario de naciones". De ahí la presencia obligada de todos ellos en los ritos del poder que se escenifican en los Parlamentos (ver Foerster 1993 y Zabala 1998, 2008).

Los Parlamentos tienen la virtud de ser una institución barroca (un ritual) que permite que a pesar de la innegable ontological rupture que verifica Dillehay, el pacto resulte posible. Se trata, por supuesto, más del pacto en torno a un middleground que a una solución política específica y definitiva. Es la sabiduría barroca jesuita frente al malestar ilustrado de un Vicuña Mackenna que no puede aceptar un pacto de esa naturaleza, donde los mapuche aparecen como "señores" a los cuales hay que "tributar". Es posible que Vicuña Mackenna tuviera en mente la amarga queja de los militares de la frontera, opuestos a la política de los parlamentos y uno de los discursos de Lautaro reproducidos en el Canto XII de La Araucana donde el longko exige:

Treinta mujeres vírgenes apuestas por tal concierto habéis de dar cada año, blancas, rubias, hermosas, bien dispuestas, de quince años a veinte, sin engaño. Han de ser españolas, y tras estas, treinta capas de verde y fino paño, y otras treinta de púrpura tejidas, con fino hilo de oro guarnecidas.

También doce caballos poderosos, nuevos y ricamente enjaezados, domésticos, ligeros y furiosos...

Porque Dillehay no tiene conciencia del Pacto Colonial, y cree ver o guerra o política, deja de lado el vínculo indisoluble entre ambas, y no dimensiona el impacto radical de la "Pacificación de la Araucanía". Así ve pura continuidad donde hay discontinuidad:

Prior to the arrival of the Spanish, their past political organization was characterized by small to large cacicazgos or chiefdoms, except in times of war against the Spanish and later the Chileans when more formal, semi-centralized polities headed by guentoqui war leaders were formed to defend their lands. It was not until the end of the XIX century that the Mapuche were finally defeated by the Chilean army and confined to reducciones.

Para nosotros la "Pacificación" implicó el fin de la frontera y de lo que hemos llamado Pacto Colonial y la emergencia de un nuevo y precario arreglo: una suerte de "Pacto Reduccional", donde nuevamente la cuestión de la soberanía se redefinió. Víctor Toledo Llancaqueo fue el primero en insistir en una lectura de este tipo de la ley de radicación de 1866, y cómo su aplicación efectiva posterior traicionó lo "pactado". Esa será también la visión de la dirigencia mapuchehuilliche a lo largo del siglo XX (desde Manuel Aburto Panguilef a José Quidel), así como la de los diputados mapuche (desde Francisco Melivilu a Venancio a Coñuepan) (ver Foerster y Montecino 1988; Menard 2013 y Vergara 2005). Solo esta perspectiva da sentido a la "deuda histórica" que actualmente postulan los movimientos mapuches respecto del Estado de Chile.

El "Pacto Reduccional" y su efecto más visible, las casi tres mil "reducciones" que se crearon a raíz del proceso de radicación a comienzos del siglo XX, fue entendido por el Estado y la elite chilena como transitorio, como un puente para la integración de los mapuches (al "Estado-Nación") y de sus tierras (al "Mercado"). En cambio, la sociedad mapuche valoró la "reducción" en su merito, porque le permitió recrearse, impulsando y transformado sus mecanismos de sociabilidad (parentesco, liderazgo, etc.), así como su comunidad ritual (esta constatación fue la gran contribución de Faron a los estudios mapuche). Para la sociedad mapuche la defensa de las tierras reduccionales (el territorio) ha pasado siempre por una apelación al Estado en el cumplimiento del pacto que dio origen a las reducciones. Este reclamo supuso un involucramiento de la dirigencia mapuche con el sistema legal (la búsqueda de la mejor ley), así como la "colonización" del Estado (como podemos ver en el proyecto de la Corporación Araucana: la DASIN; y como lo ha sido en el gran proyecto de las organizaciones de los años 1980: la CONADI) (ver Vergara et al. 2005). Desde esta discontinuidad, tanto el Pacto Colonial como el Pacto Reduccional tienen un punto en común: la política sigue siendo 
el escenario privilegiado de procesamiento de la alteridad y de la identidad.

A partir de la década de 1990 el "Pacto Reduccional" fue puesto en cuestión. Primero, por la organización Aukiñ Wallmapu Ngulam, encabezada por Aucán Huilcamán, al demandar las "tierras ancestrales" (las que no estaban consideradas en los Títulos de Merced). Posteriormente, por la Coordinadora Arauco Malleco, con la promoción y creación de "territorios liberados", lo que equivale a romper con la mediación estatal que se encuentra en el núcleo del Pacto Reduccional. Todo esto en un escenario internacional repentinamente sensible a los "derechos indígenas" (Convenio 169, etc.), que convive con un escenario nacional y con una ley indígena que por primera vez reconocía las "etnias" dando continuidad a la política de mediación del Estado (así la CONADI emerge como un híbrido estatal-indígena). Sin embargo, esta convergencia positiva hacia la demanda indígena choca con un escenario regional en el que la reconversión productiva de la Araucanía, de zona triguera a grandes empresas forestales, provoca una crisis de sustentabilidad económica y medioambiental de las comunidades rurales que, a comienzos del siglo XXI, siguen siendo el núcleo identitario y social en el que se construye la soberanía mapuche (ver Foerster 1999; J. Marimán 2012; P. Marimán 1997a, 1997b, 2011; P. Marimán et al. 2006; Pairicán 2014).
En este contexto el tema de la soberanía es central, pero también complejo, ya sea por la tradición unitaria de la cultura política chilena, ya sea porque el salto político demandado por la CAM no logra generar una sensibilidad en la comunidad más allá de sus intereses directos y locales. Esto recuerda lo que Dillehay describe como la regional non-centralization característica de la armadura social de la soberanía ente los mapuche. En la década de 1970 fue Thomas Melville (1976) el primero en mostrar el apego de los mapuches a una forma de organizar el poder que, si en el pasado fue funcional al sistema de frontera, bajo el dominio colonial republicano los limitaba seriamente en sus luchas políticas; recientemente Magnus Course (2005) han vuelto a este asunto al insistir en que el mundo rural mapuche no se deja necesariamente capturar por las exigencias de las organizaciones políticas urbanas.

Se podría pensar, y el texto de Dillehay ayuda a ello, que nos enfrentamos a una coyuntura histórica que alude claramente a la emergencia de un nuevo contexto postreduccional. ¿Significará esto la emergencia de un nuevo pacto? Creemos que esa pregunta es la que anima en nuestros días el antiguo escenario interétnico de procesamiento de la soberanía que llamamos impropiamente "el conflicto mapuche". Un escenario, como propone Boccara, anterior a la emergencia de lo mapuche. Y, como muestra Dillehay, un escenario ciertamente más viejo que Chile.

\section{Referencias Citadas}

Bengoa, J. 1985. Historia del Pueblo Mapuche, Siglo XIX y XX. Ediciones SUR, Santiago.

Bengoa, J. 2003. Historia de los Antiguos Mapuches del Sur. Desde Antes de la llegada de los Españoles hasta las Paces de Quilín. Catalonia, Santiago.

Boccara, G. 2007. Los vencedores. Los mapuche en la época colonial, IIAM (Instituto de Investigaciones Arqueológicas y Museo), Universidad Católica del Norte.

Course, M. 2005 Mapuche Person, Mapuche People. Individual and Society in Indigenous Southern Chile. London School and Political Science, London.

Dillehay, T.D. 2011 Monumentos, Imperios y Resistencia en Los Andes: El Sistema de Gobierno Mapuche y sus Narrativas. Ocho Libros, Santiago.

Faron, L. 1956. Araucanian Patri-Organization and the Omaha System. American Anthropologist. LVIII(3):435-456.
Faron, L. 1969. Los Mapuches, su Estructura Social. Instituto Indigenista Interamericano, México, D.F.

Foerster, R. 1993. Jesuitas y Mapuches: 1593-1767. Editorial Universitaria, Santiago.

Foerster, R. 1999. Movimiento étnico o movimiento etno-nacional mapuche. Revista de Crítica Cultural 18:52-58.

Foerster, R. y S. Montecino 1988. Organizaciones, Líderes y Contiendas Mapuches: 1900-1970, CEM-PEMCI, Santiago.

Isla, J. 2006. Un lugar en el mundo. Notas pewenche para una sociología de la distancia. Manuscrito en posesión del autor.

León, L. 1992. El pacto colonial hispano-araucano y el Parlamento de 1692. Nütram 30:27-53.

Marimán, J. 2012. Autodeterminación. Ideas Políticas Mapuche en el Albor del Siglo XXI, LOM, Santiago.

Marimán, P. 1997a.Tierra y legislación indígena: una mirada desde el programa del movimiento mapuche (1910-1970). Liwen 4:143-171. 
Marimán, P. 1997b. Coñuepán en el Parlamento de 1947. Argumentos y propuestas de la Corporación Araucanía. Liwen 5:157-175.

Marimán, P. 2011. La República y los mapuches: 1819-1828. En Ta in fijke xipa rakizuameluwün. Historia y Colonialismo y Resistencia desde el País Mapuche, pp. 65-88. Ediciones Comunidad de Historia Mapuche, Santiago.

Marimán, P., S. Caniuqueo, J. Millalén y R. Levil 2006. i...Escucha, winka...! Cuatro ensayos de Historia Nacional Mapuche y un epílogo sobre el futuro, LOM, Santiago.

Melville, T. 1976. The Nature of Mapuche Social Power. Tesis doctoral, The American University, Washington DC.

Menard, A. 2013. Estudio preliminar, notas y edición al Libro Diario del Presidente de la Federación Araucana, 1940, 1942, 1948-1951. Manuel Aburto Panguilef, Colibris Ediciones, Santiago.

Pairicán, F. 2014. Malón, La Rebelión del Movimiento Mapuche 1990-2013. Pehuén, Santiago.

Toledo, V. 2001. En segura y perpetua propiedad. Notas sobre el debate jurídico sobre derechos de propiedad indígena en Chile, siglo XIX. Actas $4^{\circ}$ Congreso Chileno de Antropología,
Tomo II, pp. 1129-1136. Colegio de Antropólogos de Chile, Santiago.

Vergara, J.I. 2005. La Herencia Colonial del Leviatán. El Estado y los Mapuche-Huilliche (1750-1881), Centro de Investigaciones del Hombre del Desierto (CIHDE) y Ediciones Instituto de Estudios Andinos, Universidad Arturo Prat, Iquique.

Vergara, J.I., R. Foerster y H. Gundermann 2005. Instituciones mediadoras, legislación y movimiento indígena. De la DASIN a la CONADI (1953-1954). Atenea 491:71-85.

Villalobos, S. 1995. Vida Fronteriza en la Araucanía. El Mito de la Guerra de Arauco. Editorial Andrés Bello, Santiago.

Villalobos, S., C. Aldunate, H. Zapater, L. Méndez Bertrán y C. Bascuñán 1982. Relaciones Fronterizas en la Araucanía. Ediciones Universidad Católica de Chile, Santiago.

Zavala, J. 1998. L'envers de la "Frontière" du royaume du Chili. Le cas des traités de paix hispano-mapuches du XVIIIe siècle. Histoire et Société de l'Amérique Latine 7:185-208.

Zavala, J. 2008. Los Mapuches del Siglo XVIII. Dinámica Interétnica y Estrategias de Resistencia. Editorial Universidad Bolivariana, Santiago.

\section{Notas}

1 También hay que considerar los problemas planteados por Bengoa (2003) en torno a las consecuencias del derrumbe demográfico a fines del siglo XVI y la transformación de la sociedad mapuche de "ribereña" en "ganadera".

2 Aunque en este contexto nos cuesta mucho comprender una consideración como la siguiente:

The Araucanians were (and the Mapuche still are) a patrilineal, patrilocal, and bilateral society that recognized its kin on both the mother and father's sides. ¿Significa esto que Dillehay cuestiona la orientación matrilateral que han descrito múltiples autores como la clave de la alianza y la afinidad entre los mapuche? Si este es el caso, ¿en qué materiales se apoya para proponer esta nueva descripción bilateral?

3 "Lo que se establece entre los siglos XVII y XVIII constituye una nueva tecnología de poder que tiene como principal objetivo normalizar, contabilizar, disciplinar (...) lo que emerge durante esta nueva etapa histórica es otra manera de hacer la guerra, una guerra silenciosa: la política. Y qué más eminentemente político que las grandes reuniones hispano-mapuche, llamados parlamentos (Boccara 2003:254). 
